\title{
Effectiveness and Safety of Apixaban versus Warfarin in Venous Thromboembolism Patients with Chronic Kidney Disease
}

\author{
Alexander T. Cohen ${ }^{1}$ Janvi Sah ${ }^{2}$ Amol D. Dhamane ${ }^{3}$ Theodore Lee ${ }^{4}$ Lisa Rosenblatt $^{3}$ \\ Patrick Hlavacek $^{4}$ Birol Emir $^{4}$ Rachel Delinger ${ }^{2}$ Huseyin Yuce $^{5}$ Xuemei Luo $^{6}$
}

${ }^{1}$ Department of Hematological Medicine, Guy's \& St. Thomas' NHS Foundation Trust, King's College London, London, United Kingdom

2 SIMR, LLC, Ann Arbor, Michigan, United States

${ }^{3}$ Bristol Myers Squibb Company, Lawrenceville, New Jersey, United States

${ }^{4}$ Pfizer Inc., New York, New York, United States

${ }^{5}$ Department of Mathematics, New York City College of Technology, City University of New York, New York, New York, United States

6 Pfizer Inc., Groton, Connecticut, United States

Address for correspondence Alexander T. Cohen, MD, Department of Hematological Medicine, Guy's and St. Thomas' Hospitals, King's College London, London WC2R 2LS, United Kingdom (e-mail: alexander.cohen@kcl.ac.uk).

Thromb Haemost 2022;122:926-938.

\begin{abstract}
Keywords

- chronic kidney disease

- apixaban

- venous thromboembolism

There has been limited evidence reported about the outcomes of oral anticoagulants among patients with venous thromboembolism (VTE) and chronic kidney disease (CKD), especially those with stage $V /$ end-stage renal disease (ESRD). This retrospective cohort analysis of five U.S. claims databases evaluated the risk of recurrent VTE, major bleeding (MB), and clinically relevant nonmajor bleeding (CRNMB) for apixaban versus warfarin among VTE patients diagnosed with CKD, including ESRD. Inverse probability treatment weighting (IPTW) was used to balance patient characteristics between treatment cohorts. Hazard ratios (HRs) were calculated for recurrent VTE, MB, and CRNMB among patients with CKD who experienced an index VTE. An interaction analysis was conducted to evaluate treatment effects across different stages of CKD. A total of 29,790 VTE patients with CKD were selected for analyses, of whom 10,669 (35.8\%) initiated apixaban and 19,121 (64.2\%) initiated warfarin. Among IPTWbalanced patient cohorts, the apixaban group had significantly lower risk of recurrent VTE (HR: 0.78; 95\% confidence interval [Cl]: 0.66-0.92), MB (HR: 0.76; 95\% Cl: 0.650.88 ), and CRNMB (HR: $0.86 ; 95 \% \mathrm{Cl}: 0.80-0.93$ ) than the warfarin group. When stratified by CKD stage (stage I/II: $8.2 \%$; stage III: $49.4 \%$; stage IV: $12.8 \%$; stage V/ESRD: $12.0 \%$; stage unspecified: $17.6 \%$ ), no significant interaction was observed for effects of apixaban versus warfarin on recurrent VTE or MB. In summary, apixaban was associated with a significantly lower risk of recurrent VTE and MB than warfarin among VTE patients with CKD. CKD stages did not have significant impact on treatment effects for recurrent VTE and MB.
\end{abstract}

received

April 12, 2021

accepted after revision

October 10, 2021

published online

December 28, 2021
DOI https://doi.org/

$10.1055 / \mathrm{s}-0041-1740254$. ISSN $0340-6245$.

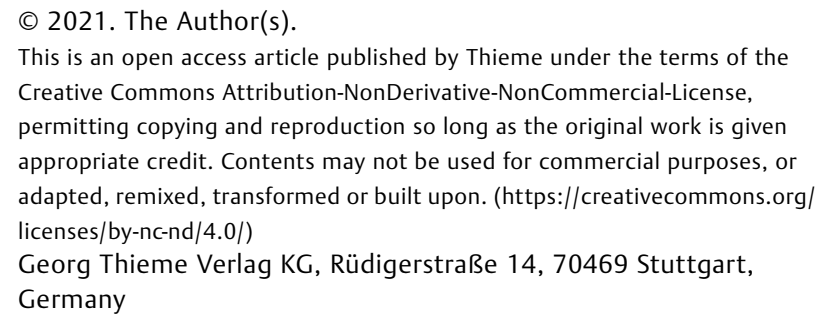




\section{Introduction}

Epidemiological studies conducted over several decades have found that venous thromboembolism (VTE) is a highly prevalent condition with $>100,000$ yearly deaths in the United States. ${ }^{1}$ Over the last decade, accumulating evidence has suggested that patients with chronic kidney disease (CKD) are at an increased risk of VTE. ${ }^{2-5}$ Decreased renal function also increases the risk of bleeding, ${ }^{6,7}$ which is associated with platelet dysfunction-particularly in advanced stages of disease. ${ }^{8,9}$ Hence, treatments with anticoagulants in VTE patients with CKD need to strike an optimal balance between preventing thrombosis and limiting hemorrhage. The American College of Chest Physicians (CHEST) and American Society of Hematology (ASH) both recommend direct oral anticoagulants (DOACs) over vitamin $\mathrm{K}$ antagonists (VKAs) for most VTE patients due to less bleeding in randomized clinical trials (RCTs) and greater convenience for patients. ${ }^{10,11}$ However, CHEST guidelines have recommended VKAs over DOACs if creatinine clearance $(\mathrm{CrCl})$ is $<30 \mathrm{~mL} / \mathrm{min}$, while the ASH guidelines have noted that the recommendation of DOACs over VKAs is not applicable to patients with $\mathrm{CrCl}<30 \mathrm{~mL} / \mathrm{min}^{10,11}$

There is limited evidence about the effects of DOACs among VTE patients with CKD. The evidence is especially lacking for those with severe $\mathrm{CKD}$, such as end-stage renal disease (ESRD), as pivotal trials of DOACs excluded VTE patients with severe renal impairments $(\mathrm{CrCl}<25 \mathrm{~mL} / \mathrm{min}$ for apixaban and $<30 \mathrm{~mL} / \mathrm{min}$ for all other DOACs ). ${ }^{11-15}$ Realworld data (RWD) (e.g., insurance claims databases) provide an additional data source to evaluate the effectiveness and safety of DOACs among VTE patients with CKD, including those with ESRD. Although several RWD studies have been conducted to evaluate the use of DOACs among VTE patients with CKD, they were limited to a single study center or a single data provider (such as the Medicare supplemental database). ${ }^{16-18}$ The aim of this study was to compare the effectiveness and safety outcomes of apixaban versus warfarin among VTE patients with CKD, including those with ESRD, using five U.S. insurance claims databases.

\section{Patients and Methods}

\section{Data Source and Patient Selection}

This study utilized data from the Centers for Medicare and Medicaid Services (CMS) fee-for-service Medicare database and four U.S. commercial claims databases: IBM MarketScan Commercial Claims and Encounter and Medicare Supplemental and Coordination of Benefits Database (MarketScan), IQVIA PharMetrics Plus (PharMetrics), Optum Clinformatics Data Mart (Optum), and the Humana Research Database (Humana).

Patients diagnosed with CKD who had $\geq 1$ medical claim for VTE in any position (index VTE event) in the inpatient or outpatient setting were identified from September 1, 2014 until the end of the study period (MarketScan: September 2018; Optum and Humana: December 2018; PharMetrics: March 2019; CMS Medicare: December 2016). Patients aged $\geq 18$ years on the index date for commercial databases or $\geq 65$ years on the index date for the Medicare database were selected if they had $\geq 1$ pharmacy claim for apixaban or warfarin during the 30-day period following the index VTE event. The date of the first warfarin or apixaban prescription was designated as the index date. International Classification of Diseases (ICD) 9th Revision, Clinical Modification (ICD-9CM) and ICD-10-CM codes were used to identify VTE (ICD-9: 415.11, 415.13, 415.19, 451.11, 451.19, 451.2, 451.81, 451.83, 451.84, 451.9, 453.1, 453.2, 453.4, 453.82, 453.83, 453.84, 453.85, 453.86, 453.87, 453.89, 453.9; ICD-10: I2692, I2699, I801, I802, I803, I809, I821, I82210, I82220, I82290, I8240, I8241, I8242, I8243, I8244, I8249, I824Y, I824Z, I8260, I8262, I82A1, I82B1, I82C1, I82890, I8290) and CKD (ICD-9: 585; ICD-10: N18). The ICD codes for VTE and CKD have been validated in previously published literature. ${ }^{19-23}$ Additional selection criteria are listed in - Fig. 1.

The baseline period was defined as 6 months prior to and including the index date. Patients' follow-up information was collected from the day after the index date through the earliest of the end of the subsequent 6-month period, index therapy discontinuation, switch to another oral anticoagulant or parenteral anticoagulant, health plan disenrollment, death, or the end of the study period. Our study employed an on-treatment approach; thus, we did not include events that occurred after a patient switched or discontinued the index treatment. Patients with each stage of CKD were identified and selected for analysis in this study by using ICD-9-CM or ICD-10-CM codes. ${ }^{24}$ Patients with CKD were stratified as stages I/II, III, IV, stage V/ESRD, or stage unspecified.

\section{Outcome Measures}

Our analysis evaluated recurrent VTE, major bleeding (MB), and clinically relevant nonmajor bleeding (CRNMB). ${ }^{25-29}$ VTE and MB were defined by primary/first-listed diagnosis in the inpatient setting. CRNMB was identified by either an inpatient admission with a secondary diagnosis code for noncritical sites of bleeding (excluded if MB occurred before the CRNMB or during the same hospitalization) or by a diagnosis code for gastrointestinal bleeding or other selected noncritical site of bleeding in the outpatient setting. This approach aligns with the current recommendation of the International Society on Thrombosis and Hemostasis. ${ }^{30}$

\section{Statistical Methods}

VTE-related variables were measured on the index VTE event date. $^{31}$ To balance patient characteristics between the treatment cohorts of VTE patients with CKD, we used stabilized inverse probability treatment weighting (IPTW). ${ }^{32}$ Covariates included in the model were demographics, clinical characteristics, and VTE-related variables. The IPTW weights were stabilized by multiplying the original weights with a constant that was equal to the expected value of being in the treatment or comparison cohorts. ${ }^{33-35}$ After IPTW, the baseline characteristics were well balanced across the cohorts and databases. The risk of recurrent VTE, MB, and CRNMB was evaluated with Cox proportional hazard models. 


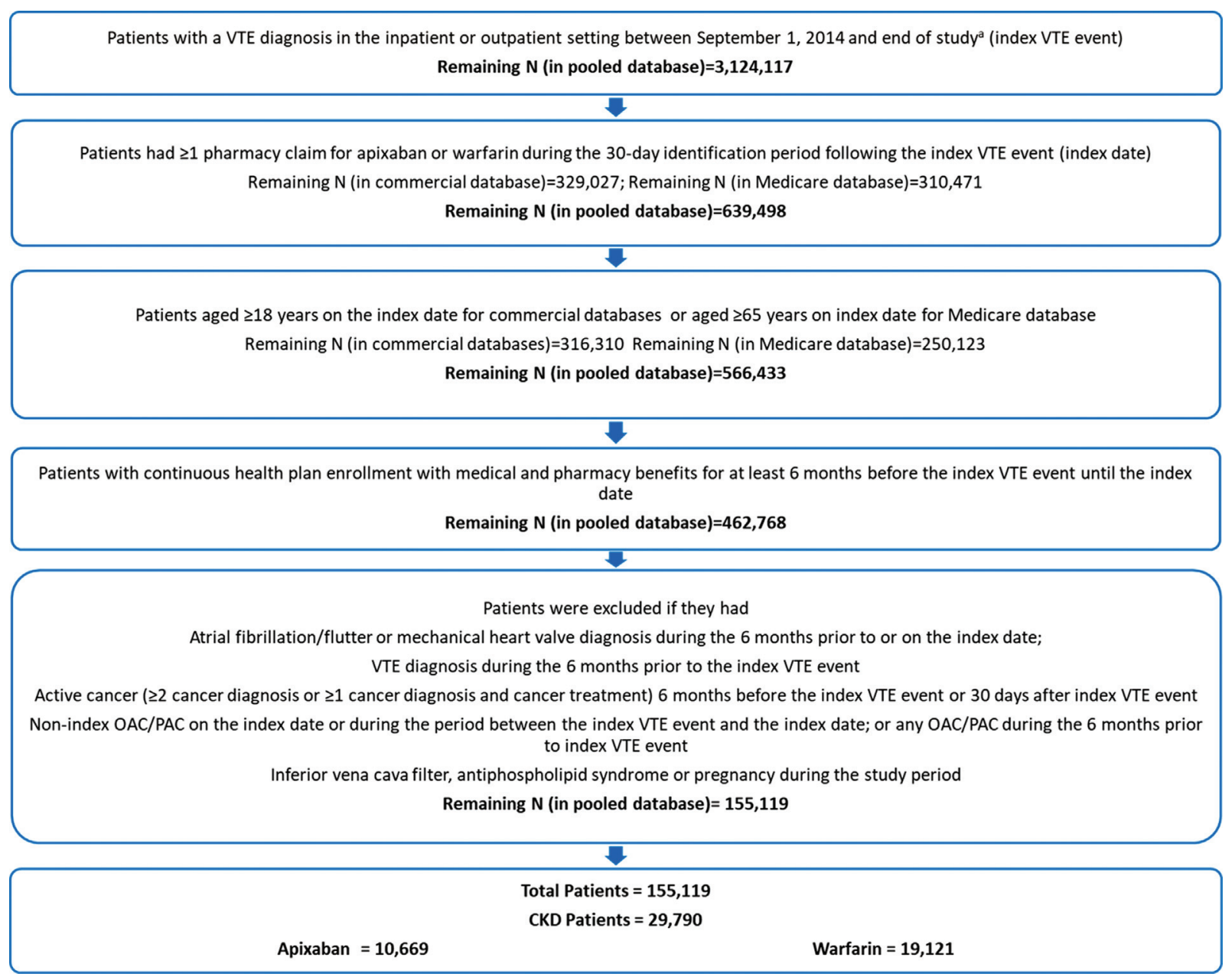

Fig. 1 Patient selection criteria. The figure illustrates the process and selection criteria used to identify patient records from the databases used in this study. Patient records were included if the patient had both CKD (defined by ICD-9-CM codes 585.1-585.9 or ICD-10-CM codes N18.1N18.9) and a diagnosis of VTE, and was treated with either apixaban or warfarin within 30 days after the index VTE. Patients were excluded on the basis of the following criteria: age (Medicare only), health insurance continuous enrollment status, medical history, and medication use. CKD, chronic kidney disease; CM, clinical modification; ICD, International Classification of Diseases; OAC, oral anticoagulant; PAC, parenteral anticoagulant; VTE, venous thromboembolism. ${ }^{a}$ MarketScan: September 2018; Optum and Humana: December 2018; PharMetrics: March 2019; CMS Medicare: December 2016.

\section{Analysis of Patients Stratified by Stage of CKD}

An interaction analysis was conducted to evaluate the treatment effect across different stages of CKD: stages I/II, III, IV, V/ESRD. Patients with stage-unspecified CKD were not included in the interaction analysis due to the lack of clinical information regarding the stage/severity of their disease. We determined the statistical significance $(p<0.10)$ of the interaction between CKD stages and treatment effects of apixaban versus warfarin and evaluated the impact of any interaction on effectiveness and safety.

\section{Results}

A total of 29,790 VTE patients with CKD were identified after application of the selection criteria, of whom 10,669 (35.8\%) initiated apixaban and 19,121 (64.2\%) initiated warfarin (-Fig. 1). The pre- and post-IPTW baseline characteristics among VTE patients with CKD who initiated apixaban or warfarin are shown in - Table 1. Before IPTW, the average Charlson comorbidity index (CCI) score was 5.2 to 5.3 , the average age was approximately 75 years, and 43.2 to $45.0 \%$ of patients were male. During the baseline period, patients initiating apixaban had a lower proportion with stage V/ESRD (8.5 vs. 13.9\%) compared with warfarin patients. After applying IPTW, baseline characteristics were well balanced among VTE patients with CKD across the apixaban and warfarin cohorts. The primary effectiveness and safety outcomes of the VTE patients with CKD are shown in - Fig. 2. VTE patients with CKD who initiated apixaban had a significantly lower risk of recurrent VTE (hazard ratio [HR]: 0.78; 95\% confidence interval [CI]: 0.66-0.92), MB (HR: 0.76; 95\% Cl: 0.65-0.88), and CRNMB (HR: 0.86; 95\% CI: 0.80-0.93) compared with warfarin patients.

To gain a better understanding of the impact of CKD stage on clinical outcomes, patients were stratified by stage. - Table 2 shows the demographic and clinical characteristics of patients stratified by different stages of CKD. Among the 29,790 VTE patients with CKD in both the apixaban and warfarin cohorts, the largest proportion of patients had CKD stage III (49.4\%) followed by stage 


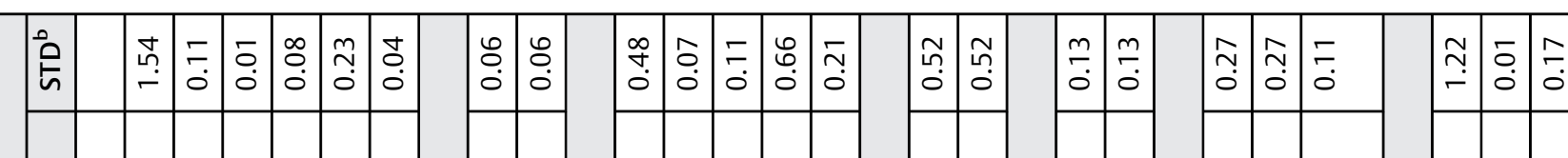

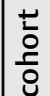

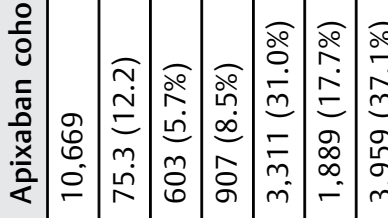

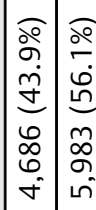

ஓㅇำ

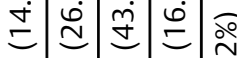

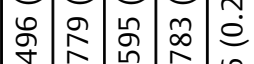

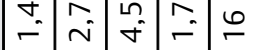

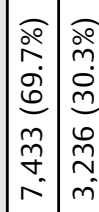

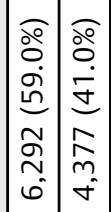

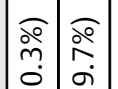

$\stackrel{2}{2} \widehat{d}$

둥

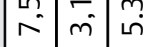

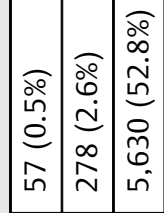

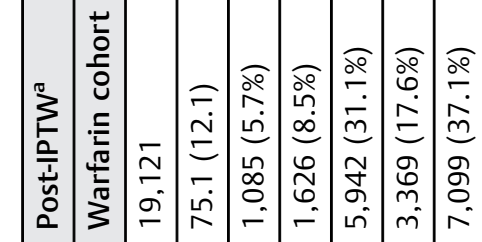

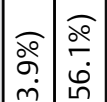

ฺे

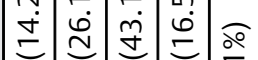

这

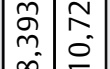

苟

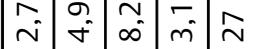

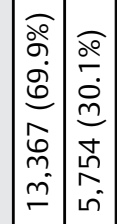

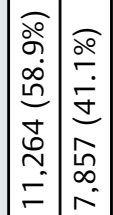

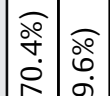

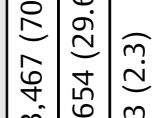

mं

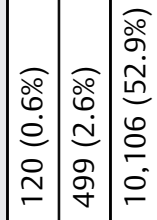

䓪

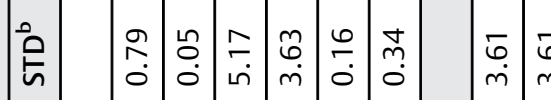

它

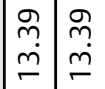

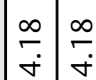

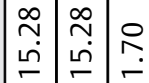

它

만.

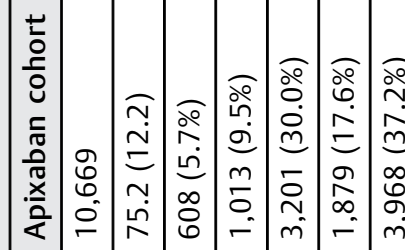

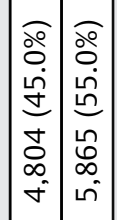

ஸे户

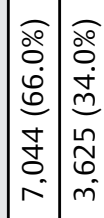

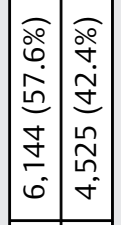

高宫

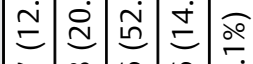

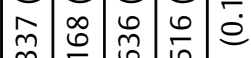

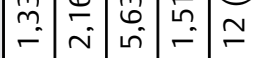

过

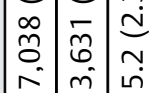

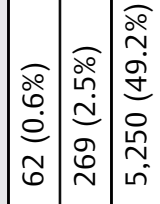

空

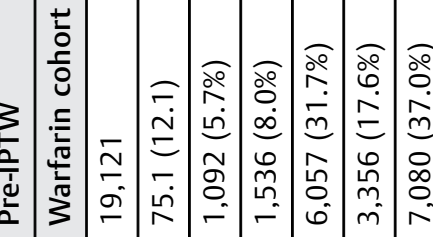

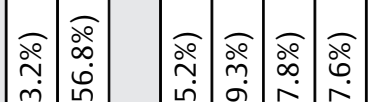

\ั.

高离

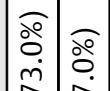

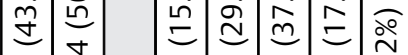

余

$\infty$

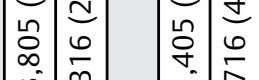

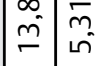

$\lesssim \approx$

苜

焉

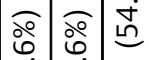

일

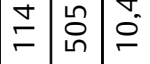

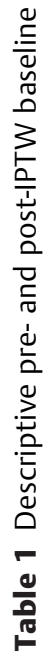

$$
+
$$

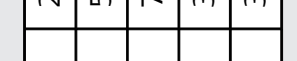

$=$

\begin{tabular}{ll}
\hline \\
\hline
\end{tabular}

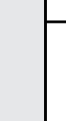

-

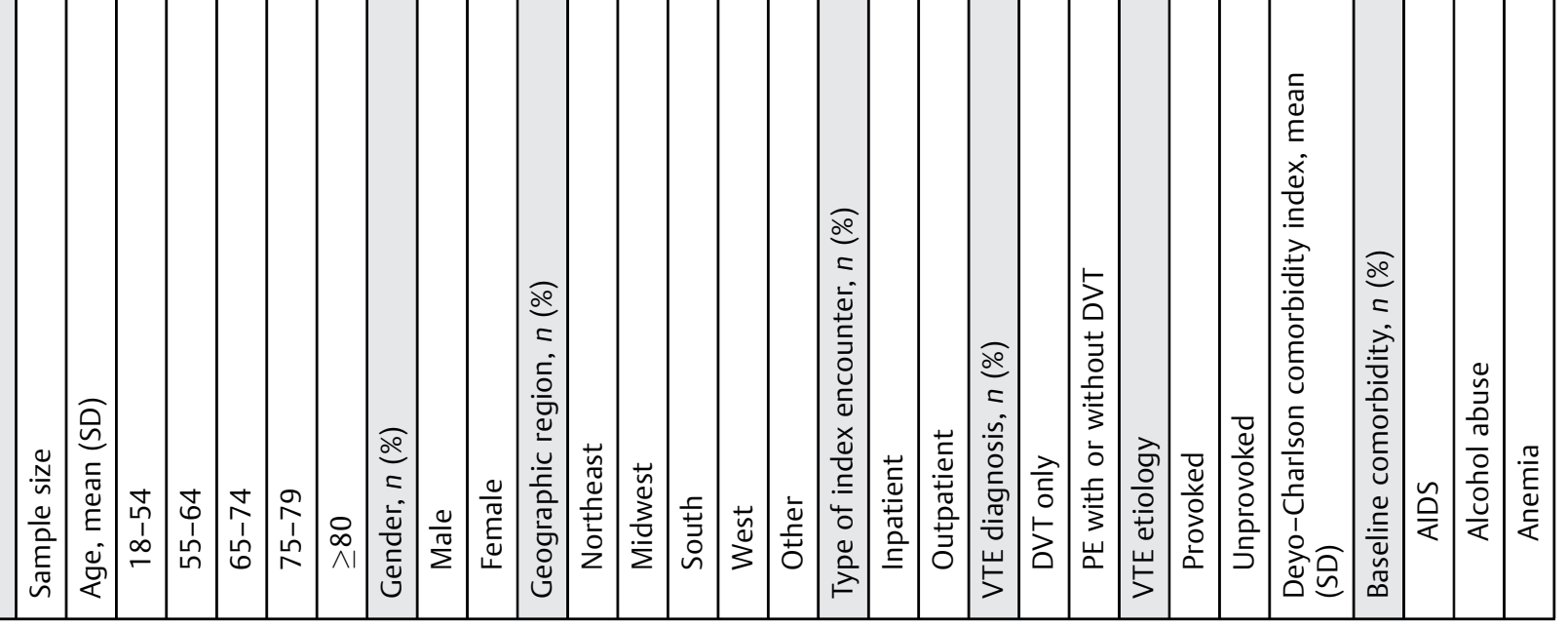




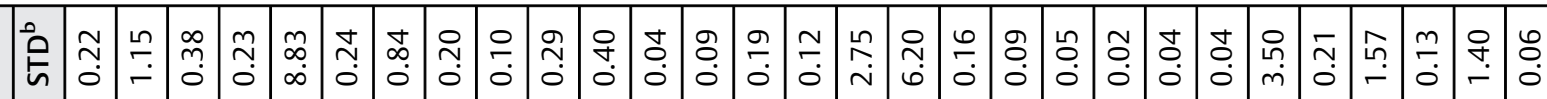

능

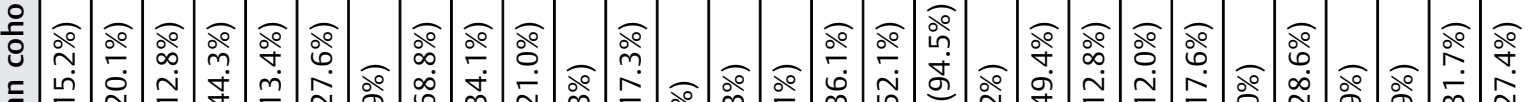

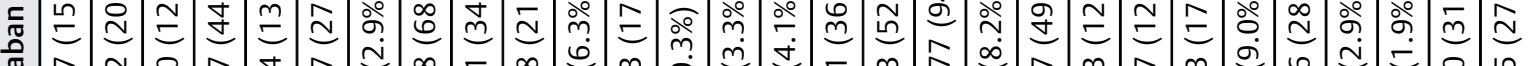

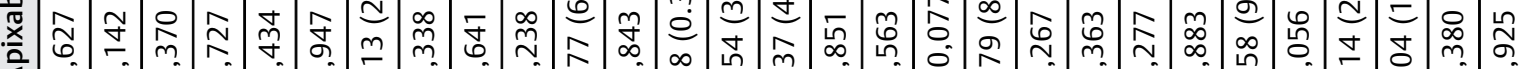

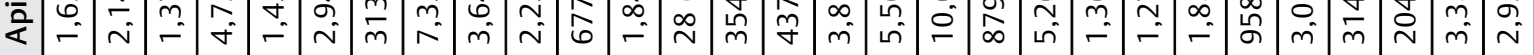

亭

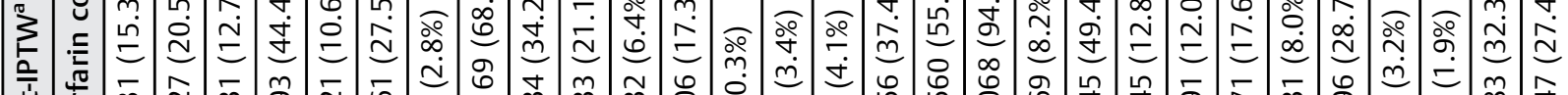

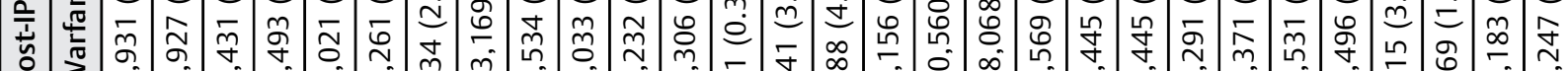

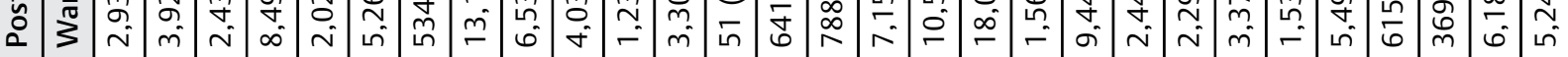

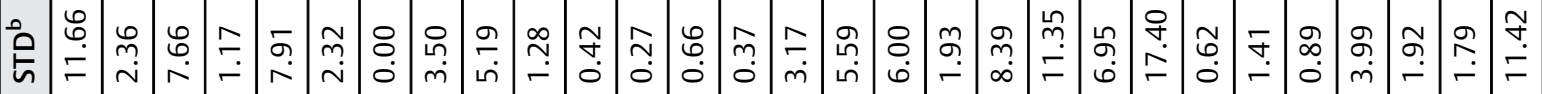

t

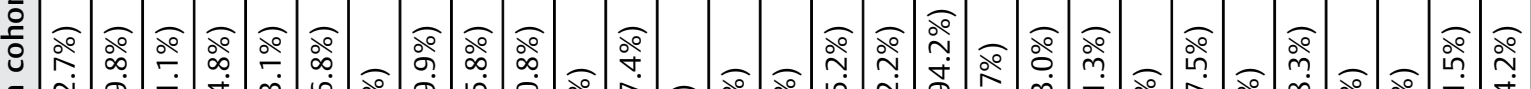

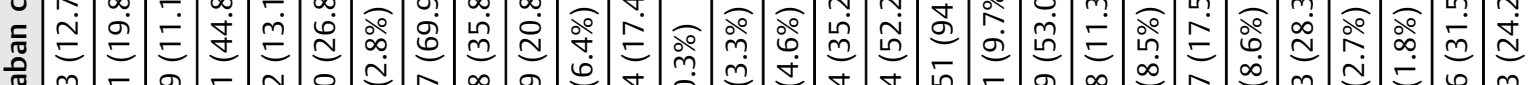

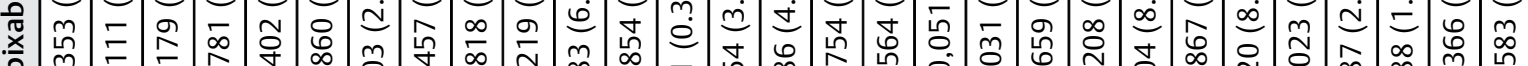

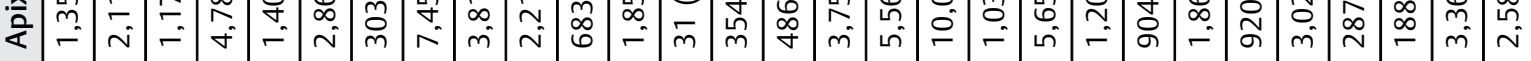

至

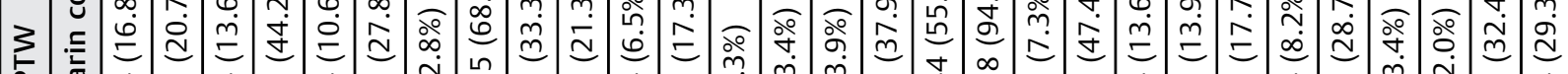
资

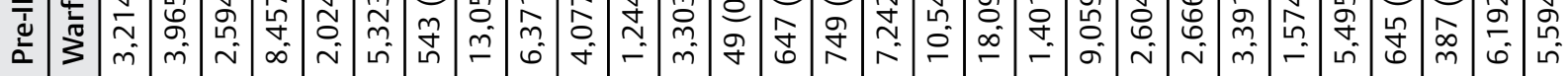

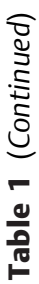

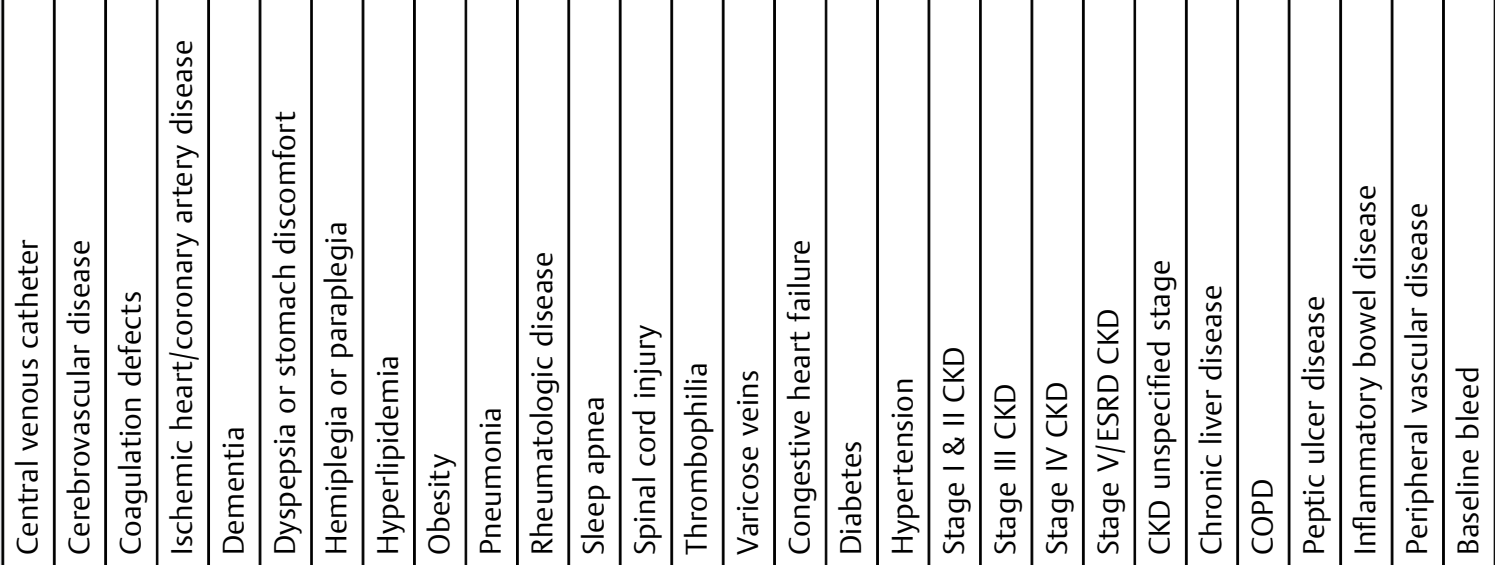




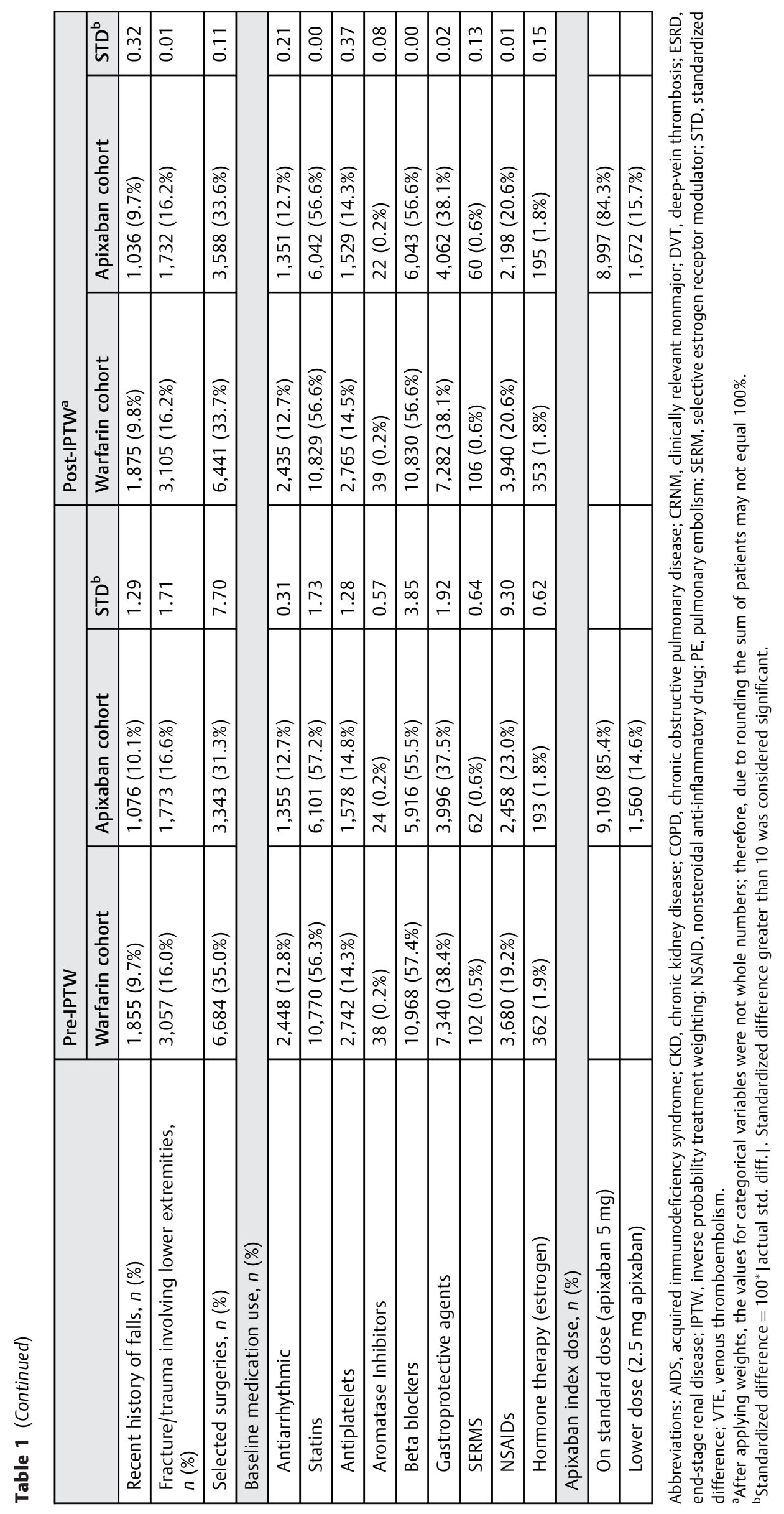




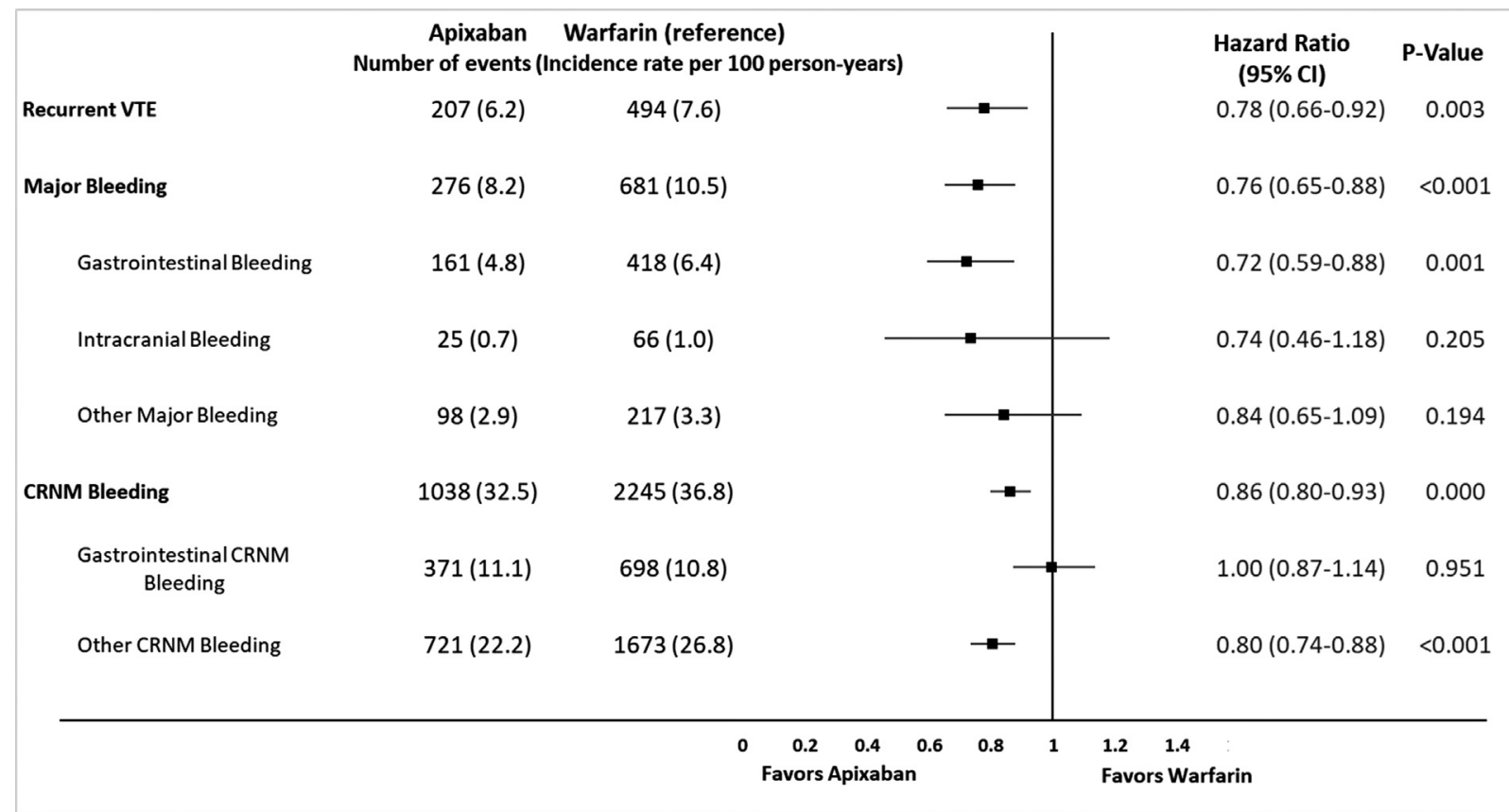

Fig. 2 Risk of recurrent venous thromboembolism (VTE), major bleeding, and clinically relevant nonmajor bleeding among patients with chronic kidney disease prescribed apixaban or warfarin to treat VTE. The figure shows a forest plot of the risks of recurrent VTE, major bleeding, and CRNMB. The patient population consisted of patients with CKD being treated for VTE with apixaban or warfarin during the study period. The number of events and the incidence per 100 person-years are listed for each outcome and treatment. Risk is indicated by an $\mathrm{HR}$ and $95 \% \mathrm{Cl}$. The degree of risk is indicated along the $x$-axis. Black squares indicate the hazard ratio; solid black lines indicate the $95 \% \mathrm{Cl}$. $\mathrm{Cl}$, confidence interval; CKD, chronic kidney disease; CRNMB, clinically relevant nonmajor bleeding; HR, hazard ratio; VTE, venous thromboembolism.

unspecified (17.6\%), stage IV (12.8\%), stage V/ESRD (12.0\%), and stage I/II (8.2\%). Patients with CKD stage IV were oldest (77.1-78.5 years), followed by stage III (76.4-76.5 years), stage unspecified (75.1-75.5 years), stage I/II (72.4-73.3 years), and stage V/ESRD (68.9 years); in contrast, the mean CCI score was the highest for stages V/ESRD (6.16.2 ) and generally decreased with the following stages (stage IV: 5.6-5.7; stage III: 5.1; stage unspecified: 5.0; stage I/II: 4.9). For type of index VTE event, the proportion of patients that had an index deep-vein thrombosis (DVT) event or provoked event was highest for stage V/ESRD patients (DVT: 76.0-77.2\%; provoked: 76.0-78.3\%) followed by stage IV (DVT: 64.5-67.4\%; provoked: 70.4-74.0\%), stage unspecified (DVT: 54.7-55.8\%; provoked: $72.2-72.6 \%$ ), stage III (DVT: 55.2\%; provoked: 68.1-68.7\%), and stage I/II (DVT: 51.5-54.2\%; provoked: 63.5-66.8\%). Other demographic characteristics with notable differences between patients with stage V/ESRD CKD and those with stage I/II CKD were a substantially greater proportion of selected surgeries (58.9-62.5 vs. $28.6-31.1 \%$, respectively) and a twofold increase in the rates of anemia (84.2-87.1 vs. 38.2-43.9\%, respectively).

-Fig. 3 shows the incidence rates and HRs of recurrent VTE, MB, and CRNMB for apixaban compared with warfarin across different stages of CKD. During the follow-up, patients with stage V/ESRD had the highest incidence rate of recurrent VTE, MB, and CRNMB ( - Fig. 3). Across all stages of CKD, patients who received apixaban had a numerically lower incidence of recurrent VTE (stage V/ESRD [7.7 vs. 11.6], stage
IV [5.8 vs. 7.8], stage III [6.2 vs. 6.9], stage I/II [5.2 vs. 7.6]) and MB (stage V/ESRD [16.9 vs. 18.0], stage IV [12.6 vs. 12.8], stage III [7.3 vs. 9.6], and stage I/II [2.9 vs. 6.6]) compared with patients who received warfarin. The apixaban group also experienced numerically lower incidences of CRNMB for patients with CKD (stage V/ESRD [38.0 vs. 52.0], stage IV [31.1 vs. 40.9], stage III [33.2 vs. 33.9], and stage I/II [28.0 vs. 33.0]).

When stratified by CKD stage, no significant interaction was observed between CKD stage and the treatment effects of apixaban versus warfarin on recurrent VTE (interaction $p=0.570$ ) or $\mathrm{MB}$ (interaction $p=0.124$ ). However, there was a significant interaction between CKD stage and treatment effects on CRNMB: while patients treated with apixaban trended toward a lower risk of CRNMB compared with patients treated with warfarin across all CKD stages, the magnitude of difference was greater for those with CKD stage IV or V/ESRD than for those with CKD stage I/II or III (-Fig. 3).

\section{Discussion}

In this retrospective analysis, which used data from five U.S. claims databases to compare the outcomes of apixaban versus warfarin among VTE patients with CKD, we found that risks of recurrent VTE, MB, and CRNMB were lower for apixaban than for warfarin. In analysis that stratified patients by different stages of CKD, no significant interaction was observed between CKD stage and the treatment 


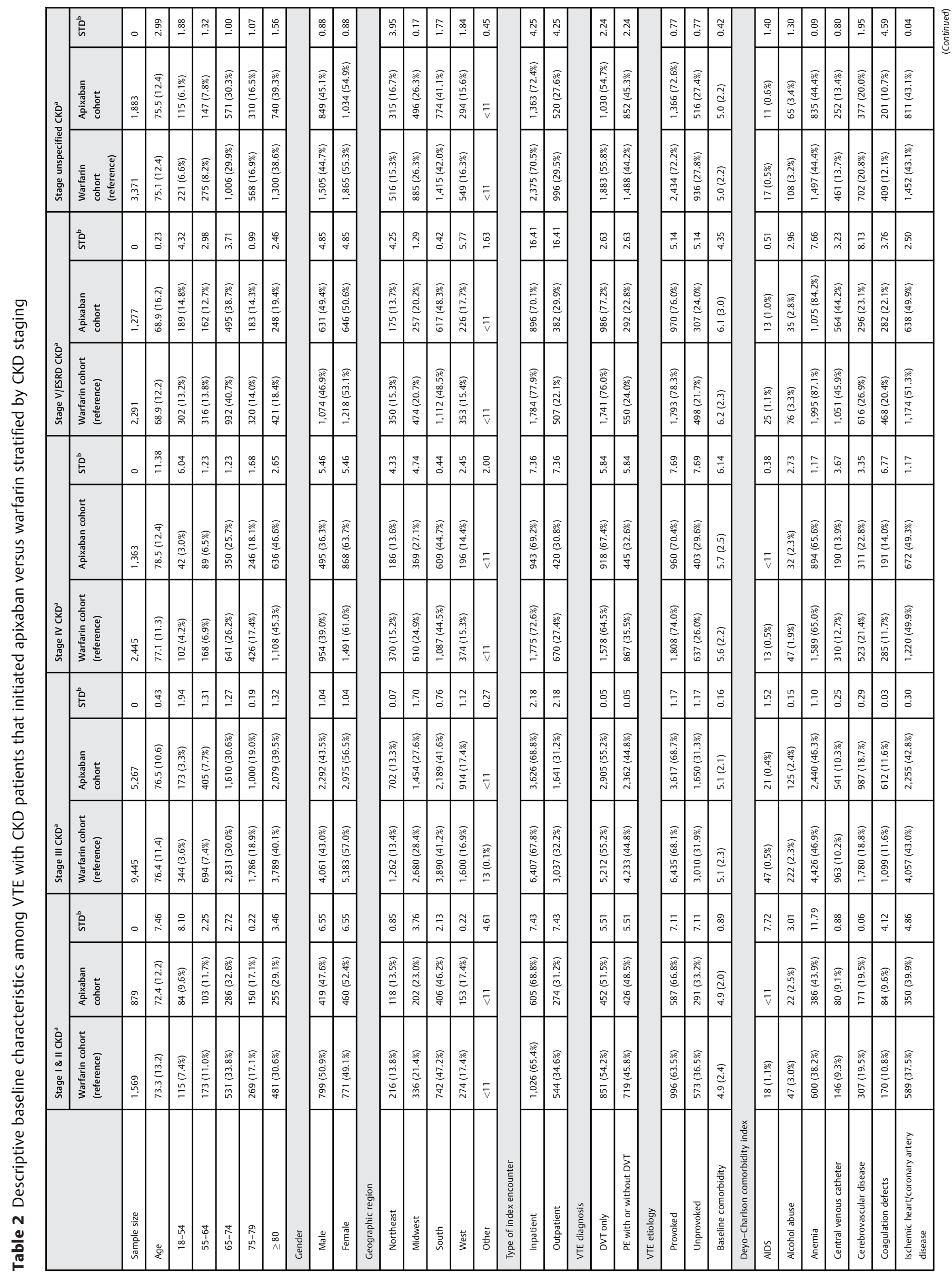




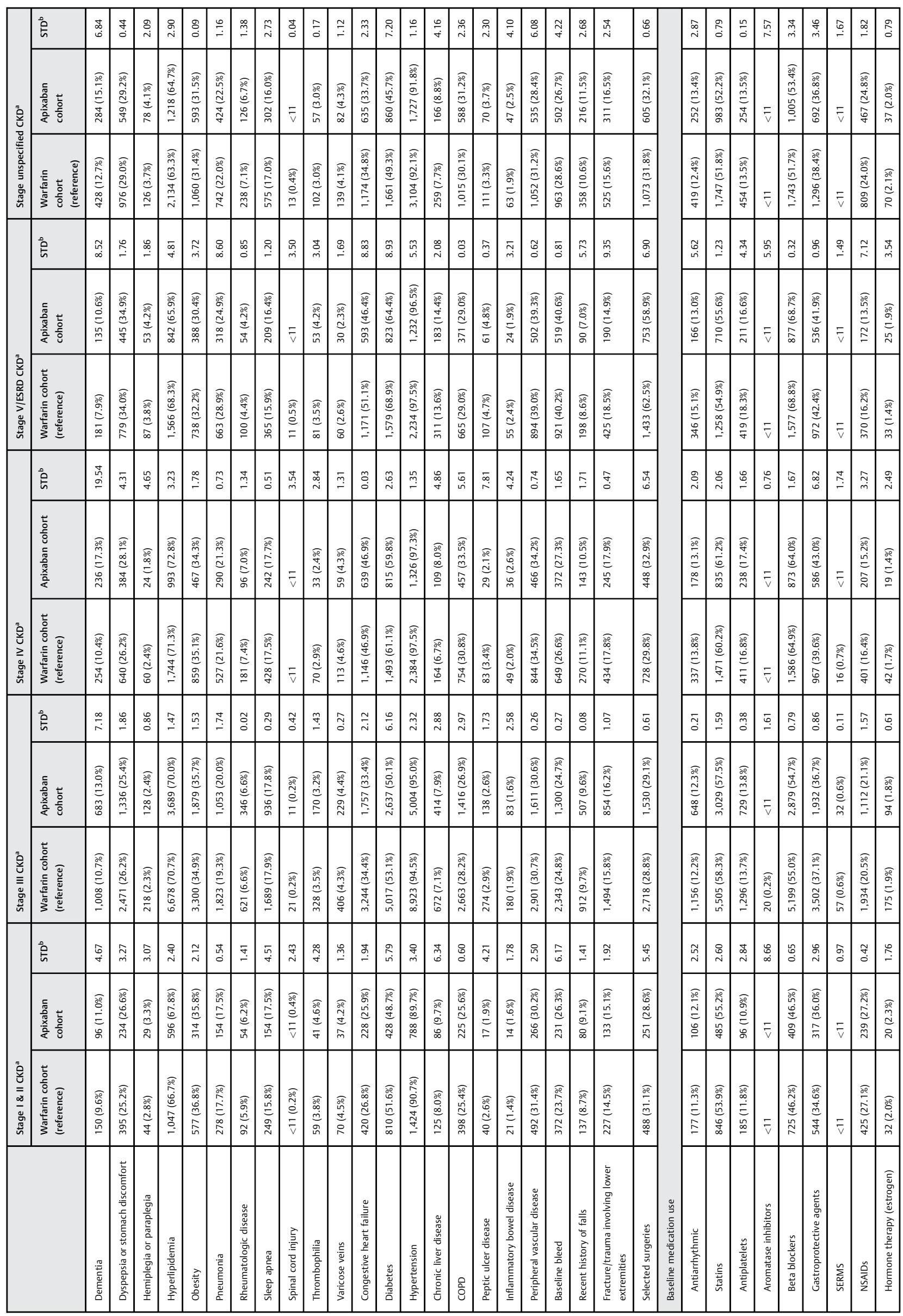




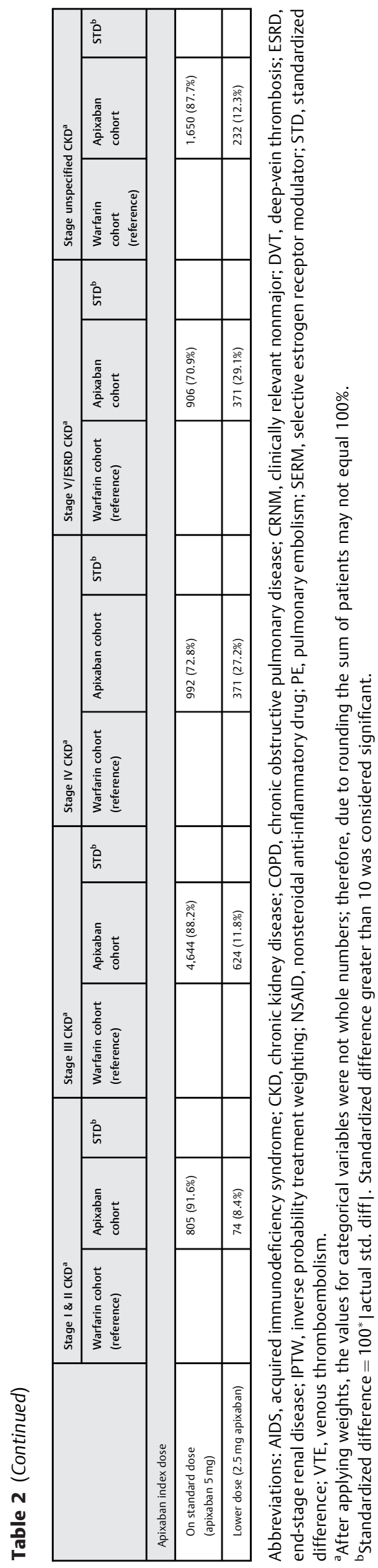

effects of apixaban versus warfarin on recurrent VTE and $\mathrm{MB}$. The lack of interaction suggests that the effects associated with use of apixaban or warfarin were consistent across different stages of CKD, including ESRD.

The findings of this RWD analysis provide complementary information to that of the AMPLIFY RCT. ${ }^{12}$ The AMPLIFY trial found that apixaban was noninferior to conventional therapy for the prevention of recurrent VTE and was associated with less bleeding. ${ }^{12}$ Moreover, included subgroup analysis showed no significant interaction between the level of renal function and treatment effects of apixaban versus warfarin on recurrent VTE (interaction $p=0.8757$ ) or MB (interaction $p=0.3606$ ), suggesting that the efficacy and safety of apixaban were consistent across the different levels of renal function. Our study expanded upon this renal subgroup analysis with additional data on effectiveness and safety of apixaban versus warfarin among VTE patients with CKD in routine clinical practice, including those with stage V/ESRD. We found apixaban to be associated with both lower risk of recurrent VTE and MB than for warfarin and consistent findings across different stages of CKD, including stage V/ESRD.

Several RWD studies have evaluated apixaban use among patients with CKD. ${ }^{16-18}$ Dawwas et al reported that recurrent VTE (8.0 vs. 13.0 per 100 person-years) and MB (7.0 vs. 15.0 per 100 person-years) were significantly lower in apixaban patients compared with warfarin patients among VTE patients with CKD. ${ }^{16}$ No significant interaction was observed between CKD status and treatment effects on recurrent VTE (interaction $p=0.90$ ); however, a significant interaction was observed for $\mathrm{MB}$ (interaction $p=0.09$ ); apixaban patients trended toward a lower risk of MB across both CKD and non-CKD cohorts. ${ }^{16}$ In a similar study of patients with $\mathrm{CrCl}<25 \mathrm{~mL} / \mathrm{min}$, Hanni et al found lower risk of thrombotic or bleeding events with apixaban (HR: 0.47; 95\% CI: 0.25-0.92) compared with warfarin. ${ }^{17}$ In addition, a systematic literature review by Cheung et al found no significant difference between apixaban and warfarin in reducing recurrent VTE or bleeding events in patients with moderate CKD as well as no difference on VTE outcomes among patients with severe CKD who were on dialysis. ${ }^{18}$ Our study extends these findings and includes a much larger sample size and more comprehensive analyses of the effectiveness and safety of apixaban versus warfarin among VTE patients with CKD. An additional strength of our study is that we evaluated not only the population of patients with CKD as a whole but also stratified them by the severity of CKD. This level of analysis can help address some of the data gaps regarding the use of anticoagulants by patients with ESRD.

This study has some limitations that are inherent to analyses using claims data. While associations can be inferred from this type of analysis, causal relationships cannot. Although IPTW has been used to balance patient characteristics between treatment cohorts, residual confounding may persist. The databases lacked information regarding inpatient bridging therapy and international normalized ratio values for the warfarin cohort, thus we could not observe 


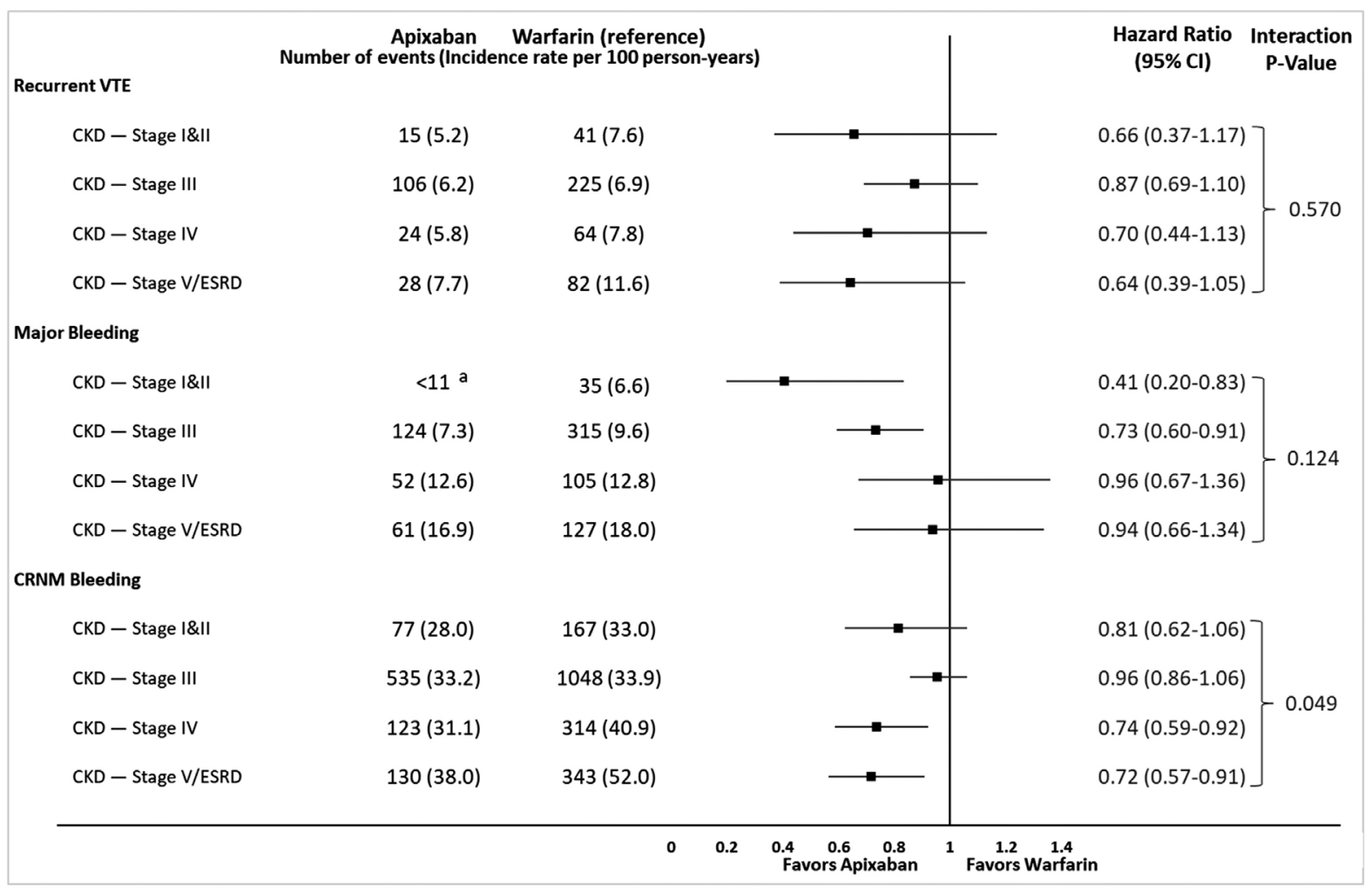

Fig. 3 Risk of recurrent venous thromboembolism (VTE), major bleeding, and clinically relevant nonmajor bleeding among patients with chronic kidney disease prescribed apixaban or warfarin to treat VTE, stratified by CKD stages. The figure shows a forest plot of the risks of recurrent VTE, major bleeding, and CRNMB. The patient population consisted of patients with CKD being treated for VTE with apixaban or warfarin during the study period, stratified by the stage of CKD (stage I/II, stage III, stage IV, stage V/ESRD). The number of events and the incidence per 100 person-years are listed for each outcome, stage, and treatment. Risk is indicated by an $\mathrm{HR}$ and $95 \% \mathrm{Cl}$. The degree of risk is indicated along the $x$-axis. The $p$-value for interaction indicates the association between CKD stages and treatment effects on a specific outcome. Black squares indicate the hazard ratio; solid black lines indicate the $95 \% \mathrm{Cl}$. $\mathrm{Cl}$, confidence interval; $\mathrm{CKD}$, chronic kidney disease; CRNMB, clinically relevant nonmajor bleeding; ESRD, end-stage renal disease; HR, hazard ratio; VTE, venous thromboembolism. a $<11$ used due to agreements with commercial providers to assure privacy for very small number of events; interaction is significant if $p<0.10$.

certain clinical treatment decisions. We identified CKD based on diagnosis codes only, without any laboratory data for renal function, and 17 to $18 \%$ of the patients had stage-unspecified CKD. In addition, patients with less severe disease, such as stage $1 / \mathrm{II}$, may be underreported. Although previous studies that validated ICD codes to identify CKD have found reasonably high positive predictive values (range: $63-97 \%$ ), ${ }^{23,36}$ results on patients stratified by CKD stages should be interpreted with caution. Both ICD-9 and ICD-10 codes were used to identify recurrent VTE and MB, yet the conversion from ICD-9 to ICD-10 may not be perfect and the presence of a code may not always be associated with adverse events (e.g., coding error or rule out criteria). The MB and recurrent VTE events were defined based on primary or first listed ICD 9/10 diagnosis codes. These events were not adjudicated based on strict clinical criteria or validated against patients' medical records. Moreover, our CRNMB definition was based on diagnosis codes without consideration of laboratory values and has not been validated through clinical confirmation. Hence, the incidence of recurrent VTE and bleeding events may be over- or underestimated. Further, we did not include death as a competing risk, as mortality data were not available for all utilized databases. Nonetheless, we expect the impact of mortality on clinical outcomes is likely to be minimal, given that our analysis of a database with available mortality data showed a low mortality rate for the study population $(\sim 5 \%)$. Finally, our patient population did not include those who were uninsured or those with other insurance types not included in our study. Thus, our results may not be generalizable to the entire population of patients with CKD and VTE.

\section{Conclusion}

Our study showed that the use of apixaban was associated with significantly lower risks of recurrent VTE, MB, and CRNMB when compared with warfarin among VTE patients with CKD. Additionally, CKD stages, including stage V/ESRD, did not have significant impacts on treatment effects for recurrent VTE and MB. While this study provides additional evidence to support the use of apixaban by VTE patients with CKD, more studies are needed to confirm our findings. 


\section{What is known about this topic?}

- Patients with chronic kidney disease (CKD) are at increased risk of venous thromboembolism (VTE) and bleeding.

- VTE patients with CKD, especially those with endstage renal disease (ESRD), were underrepresented or excluded from pivotal trials of oral anticoagulants.

- Limited studies based on a single data source showed that apixaban may be associated with a lower risk of recurrent VTE and major bleeding (MB) among patients with CKD.

\section{What does this paper add?}

- This study used five U.S. claims databases and included the largest sample to date of VTE patients with CKD being treated with apixaban versus warfarin.

- The findings of this study provide additional evidence to suggest that apixaban was associated with a reduced risk of recurrent VTE and MB among VTE patients diagnosed with CKD.

- Analysis of VTE patients with stages I to V/ESRD of CKD showed that the results on recurrent VTE and MB were consistent across different stages of CKD, including ESRD.

\section{Author Contributions}

All authors were involved in conceptualization, methodology, and writing and review of the manuscript. The statistical analysis was performed by J. Sah and R. Delinger, employees of STATinMED Research, a paid consultant to Pfizer and Bristol Myers Squibb Company.

\section{Funding}

This research was funded by Pfizer and Bristol Myers Squibb.

\section{Conflict of Interest}

A.T.C. received research support from Pfizer and Bristol Myers Squibb Company. J.S. and R.D. are employed by SIMR, LLC-a paid consultant to Pfizer and Bristol Myers Squibb Company, in connection with the development of the manuscript. T.L., P.H., B.E., and X.L. are employees of Pfizer, a study sponsor. A.D.D. and L.R. are employees of Bristol Myers Squibb Company, a study sponsor. H.Y. has no financial relationships or other potential conflicts of interest to declare.

\section{References}

1 Centers for Disease Control and Prevention. Venous thromboembolism (blood clots): data \& statistics. February 7, 2020. Accessed January 21, 2021 at: https://www.cdc.gov/ncbddd/dvt/data.html

2 Parikh AM, Spencer FA, Lessard D, et al. Venous thromboembolism in patients with reduced estimated GFR: a population-based perspective. Am J Kidney Dis 2011;58(05):746-755
3 Cheung KL, Zakai NA, Folsom AR, et al. Measures of kidney disease and the risk of venous thromboembolism in the REGARDS (Reasons for Geographic and Racial Differences in Stroke) study. Am J Kidney Dis 2017;70(02):182-190

4 Folsom AR, Lutsey PL, Astor BC, Wattanakit K, Heckbert SR, Cushman MAtherosclerosis Risk in Communities Study. Chronic kidney disease and venous thromboembolism: a prospective study. Nephrol Dial Transplant 2010;25(10):3296-3301

5 Wattanakit K, Cushman M, Stehman-Breen C, Heckbert SR, Folsom AR. Chronic kidney disease increases risk for venous thromboembolism. J Am Soc Nephrol 2008;19(01):135-140

6 Ishigami J, Grams ME, Naik RP, Coresh J, Matsushita K. Chronic kidney disease and risk for gastrointestinal bleeding in the community: the Atherosclerosis Risk in Communities (ARIC) study. Clin J Am Soc Nephrol 2016;11(10):1735-1743

7 Ocak G, Rookmaaker MB, Algra A, et al; SMART Study Group. Chronic kidney disease and bleeding risk in patients at high cardiovascular risk: a cohort study. J Thromb Haemost 2018;16 (01):65-73

8 Boccardo P, Remuzzi G, Galbusera M. Platelet dysfunction in renal failure. Semin Thromb Hemost 2004;30(05):579-589

9 Di Minno G, Martinez J, McKean ML, De La Rosa J, Burke JF, Murphy S. Platelet dysfunction in uremia. Multifaceted defect partially corrected by dialysis. Am J Med 1985;79(05): 552-559

10 Kearon C, Akl EA, Ornelas J, et al. Antithrombotic therapy for VTE disease: CHEST guideline and expert panel report. Chest 2016; 149(02):315-352

11 Ortel TL, Neumann I, Ageno W, et al. American Society of Hematology 2020 guidelines for management of venous thromboembolism: treatment of deep vein thrombosis and pulmonary embolism. Blood Adv 2020;4(19):4693-4738

12 Agnelli G, Buller HR, Cohen A, et al; AMPLIFY Investigators. Oral apixaban for the treatment of acute venous thromboembolism. $\mathrm{N}$ Engl J Med 2013;369(09):799-808

13 Bauersachs R, Berkowitz SD, Brenner B, et al; EINSTEIN Investigators. Oral rivaroxaban for symptomatic venous thromboembolism. N Engl J Med 2010;363(26):2499-2510

14 Büller HR, Décousus H, Grosso MA, et al; Hokusai-VTE Investigators. Edoxaban versus warfarin for the treatment of symptomatic venous thromboembolism. N Engl J Med 2013;369(15): 1406-1415

15 Schulman S, Kearon C, Kakkar AK, et al; RE-COVER Study Group. Dabigatran versus warfarin in the treatment of acute venous thromboembolism. N Engl J Med 2009;361(24):2342-2352

16 Dawwas GK, Smith SM, Dietrich E, Lo-Ciganic WH, Park H. Comparative effectiveness and safety of apixaban versus warfarin in patients with venous thromboembolism. Am J Health Syst Pharm 2020;77(03):188-195

17 Hanni C, Petrovitch E, Ali M, et al. Outcomes associated with apixaban vs warfarin in patients with renal dysfunction. Blood Adv 2020;4(11):2366-2371

18 Cheung CYS, Parikh J, Farrell A, Lefebvre M, Summa-Sorgini C, Battistella M. Direct oral anticoagulant use in chronic kidney disease and dialysis patients with venous thromboembolism: a systematic review of thrombosis and bleeding outcomes. Ann Pharmacother 2021;55(06):711-722

19 Tamariz L, Harkins T, Nair V. Mini-sentinel systematic evaluation of health outcome of interest definitions for studies using administrative data: venous thromboembolism report. Mini-Sentinel website. August 11, 2011. Accessed January 21, 2021 at: https:// www.sentinelinitiative.org/sites/default/files/surveillance-tools/validations-literature/Mini-Sentinel-HOI-Evidence-Review-Venous-Thromboembolism-Report.pdf

20 Fang MC, Fan D, Sung SH, et al. Validity of using inpatient and outpatient administrative codes to identify acute venous thromboembolism: the CVRN VTE study. Med Care 2017;55(12): e137-e143 
21 Chen SY, Wu N, Gulseth M, et al. One-year adherence to warfarin treatment for venous thromboembolism in high-risk patients and its association with long-term risk of recurrent events. J Manag Care Pharm 2013;19(04):291-301

22 Deitelzweig SB, Lin J, Kreilick C, Hussein M, Battleman D. Warfarin therapy in patients with venous thromboembolism: patterns of use and predictors of clinical outcomes. Adv Ther 2010;27(09): 623-633

23 Navaneethan SD, Jolly SE, Schold JD, et al. Development and validation of an electronic health record-based chronic kidney disease registry. Clin J Am Soc Nephrol 2011;6(01):40-49

24 Jalal K, Anand EJ, Venuto R, Eberle J, Arora P. Can billing codes accurately identify rapidly progressing stage 3 and stage 4 chronic kidney disease patients: a diagnostic test study. BMC Nephrol 2019;20(01):260

25 Weycker D, Li X, Wygant GD, et al. Effectiveness and safety of apixaban versus warfarin as outpatient treatment of venous thromboembolism in U.S. clinical practice. Thromb Haemost 2018;118(11):1951-1961

26 Hlavacek P, Guo JD, Rosenblatt L, et al. Safety, effectiveness, and health care cost comparisons among elderly patients with venous thromboembolism prescribed warfarin or apixaban in the United States Medicare population. Curr Med Res Opin 2019;35(12): 2043-2051

27 Weycker D, Wygant GD, Guo JD, et al. Bleeding and recurrent VTE with apixaban vs warfarin as outpatient treatment: time-course and subgroup analyses. Blood Adv 2020;4(02):432-439

28 Cohen AT, Keshishian A, Lee T, et al. Effectiveness and safety of apixaban, low-molecular-weight heparin, and warfarin among venous thromboembolism patients with active cancer: a U.S. claims data analysis. Thromb Haemost 2020;121(03):383-395

29 Cunningham A, Stein CM, Chung CP, Daugherty JR, Smalley WE, Ray WA. An automated database case definition for serious bleeding related to oral anticoagulant use. Pharmacoepidemiol Drug Saf 2011;20(06):560-566

30 Kaatz S, Ahmad D, Spyropoulos AC, Schulman SSubcommittee on Control of Anticoagulation. Definition of clinically relevant nonmajor bleeding in studies of anticoagulants in atrial fibrillation and venous thromboembolic disease in non-surgical patients: communication from the SSC of the ISTH. J Thromb Haemost 2015;13(11):2119-2126

31 Kearon C, Ageno W, Cannegieter SC, Cosmi B, Geersing GJ, Kyrle PASubcommittees on Control of Anticoagulation, and Predictive and Diagnostic Variables in Thrombotic Disease. Categorization of patients as having provoked or unprovoked venous thromboembolism: guidance from the SSC of ISTH. J Thromb Haemost 2016; 14(07):1480-1483

32 Austin PC. The use of propensity score methods with survival or time-to-event outcomes: reporting measures of effect similar to those used in randomized experiments. Stat Med 2014;33(07): $1242-1258$

33 Austin PC, Stuart EA. Moving towards best practice when using inverse probability of treatment weighting (IPTW) using the propensity score to estimate causal treatment effects in observational studies. Stat Med 2015;34(28):3661-3679

34 Thoemmes F, Ong AD. A primer on inverse probability of treatment weighting and marginal structural models. Emerg Adulthood 2016;4(01):40-59

35 Xu S, Ross C, Raebel MA, Shetterly S, Blanchette C, Smith D. Use of stabilized inverse propensity scores as weights to directly estimate relative risk and its confidence intervals. Value Health 2010; 13(02):273-277

36 Winkelmayer WC, Schneeweiss S, Mogun H, Patrick AR, Avorn J, Solomon DH. Identification of individuals with CKD from Medicare claims data: a validation study. Am J Kidney Dis 2005;46(02): $225-232$ 